\section{Seeing nuclear complexity}

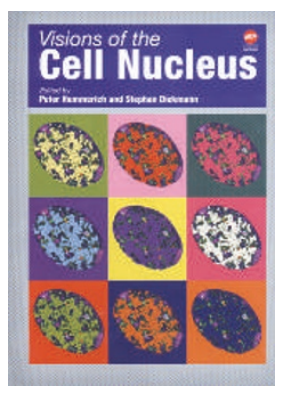

\section{Visions of the Cell Nucleus}

\section{Edited by Peter Hemmerich and Stephan Diekmann}

American Scientific Publishers • 2005

$\$ 450$

\section{Tom Misteli}

The way we do biology has changed in the last decade. We are moving away from molecularly dissecting individual events as we increasingly seek to understand the complexity of biological systems - a shift that has been heralded by the emergence of proteomics and genomics. In the face of these developments, it is easy to forget that cell biologists have always dealt with the cell's complexity by probing complicated cellular processes, often using imaging methods. Arguably, the cell nucleus is one of the most complex cellular organelles and one that has been extensively interrogated by visual methods. In Visions of the Cell Nucleus, Peter Hemmerich and Stephan Diekmann set out to capture a picture of what we have learnt about the cell nucleus since its discovery in the nineteenth century.

The term 'visions' has several meanings. It can describe a 'fleeting glimpse of an object' or an 'object in its general context at a larger scale'. The editors cleverly provide snapshots from selected areas of nuclear research to create a picture of the complexity of nuclear structure and function. To do so, they cast a wide net and include chapters on topics ranging from spatial genome organization, transcription and pre-mRNA processing, to replication, mitosis, epigenetics and nuclear bodies. The chapters are written by acclaimed experts and are of a high quality, generally very comprehensive, well written and accessible. However, as is inevitable when dealing with so many different authors, the chapters are in places imbalanced, being of variable length and depth.

As befits a book on a field of research that is dominated by imaging methods, this volume stands out for its visual appeal. Not only is the layout well designed and clear, each chapter centres around multiple high-quality colour plates, and in many chapters you can find more colour illustrations than you would usually find in an entire textbook. The editors and publishers should be commended for including these abundant visual aids; by doing so, they have raised the bar for other cell biology textbooks.

Cell biological investigations of nuclear structure have been driven by technological developments, particularly in the field of imaging. It is therefore appropriate that the final chapters cover key microscopy techniques used in nuclear studies including energy transfer, correlation spectroscopy and multiphoton microscopy. However, while these chapters will undoubtedly be useful to some readers, they are somewhat off target and do not directly deal with nuclear biology. Unfortunately, their inclusion appears to come at the expense of several important topics that are only mentioned in passing and deserve more coverage. Most prominent among them are recent developments in understanding the dynamic properties of nuclear bodies and nuclear proteins, the exploding field of lamin-related diseases, the exponential growth of live-cell imaging techniques, and the integration of genomic and proteomic methods with imaging techniques.

The lack of these topics is related to the key weakness of this collection. The book is already considerably outdated. This is most evident by the fact that the most recent citation in the book is more than three years old. In a rapidly moving field this is a significant shortcoming. A further weakness is the poor index. Although you can generally find most relevant terms, searching for them is often confusing; for example, many keywords are listed both in the singular and plural form, or both as abbreviations and in full, and, oddly, the two entries often guide the reader to two different, unrelated pages or even chapters.

\section{As befits a book on a field of research that is dominated by imaging methods, this volume stands out for its visual appeal.}

The term 'vision' can also refer to what lies ahead and what might be. While the chapters do provide a satisfying summary of the current state of knowledge, very few chapters discuss where the field is headed. This is a shame since the study of nuclear architecture is undergoing a veritable renaissance and many new avenues of investigation are emerging. Discussing more forward-looking material would have been an opportunity to communicate the current excitement in the field and to define landmarks worth striving for. Similarly, there is little mention of some of the more controversial topics in the field including the long-standing debate on the existence of a nuclear scaffold or the more recent provocative discovery of intranuclear translation. The lack of these topics makes this book less visionary than it could be.

Despite these imperfections, Visions of the Cell Nucleus does provide a good picture of the status quo in the field. Many chapters will appeal to both the uninitiated student as an introduction to the field, as well as to the expert, who will find many encyclopaedic pieces of information.

A final meaning of 'vision' is that of an 'object of the imagination'. The major contribution of this book might be to provide a solid base for future views of nuclear function. It summarizes much of the current knowledge that we need to incorporate into new models of how the complex network of nuclear functions is coordinated within the architecture of the nucleus. Whether these visions will turn out to be real or merely imaginary remains to be seen. 